- The taste perception of the tongue does not change, but there is a decline in palatal perception in postmenopausal women.

- Postmenopausal women appear to have a reduced perception of sucrose.

- This can alter eating habits, such as preference for sweeter foods.

- The dental practitioner plays a key role in the management of the oral complications of the menopause.

\title{
Evaluation of gustatory function in postmenopausal women
}

\author{
C. Delilbasi, ${ }_{1}^{1}$ T. Cehiz, ${ }^{2}$ U. K. Akal ${ }^{3}$ and T. Yilmaz ${ }^{4}$
}

\begin{abstract}
Objective The aim of this study is to evaluate the gustatory function in postmenopausal women.

Subjects and methods Whole mouth taste test and spatial taste test were administered to 20 postmenopausal women (Group 1) and 20 age-matched male subjects (Group 2). Further investigations were carried out for subjective assessment of taste alteration and nutritional differences between premenopausal and postmenopausal periods.

Results There was a significant reduction in sucrose perception and in palatal sensitivity in postmenopausal women $(p<0.05)$. Only 7 $(35 \%)$ of the female patients noticed alteration in taste perception during the postmenopausal period. Alteration in dietary habits after menopause was reported by $9(45 \%)$ patients.

Conclusion This study demonstrates that taste perception of the tongue does not change, but that there is a disorder in palatal perception in postmenopausal women. Furthermore, there is tendency to preference of sweeter foods during menopause.
\end{abstract}

\section{INTRODUCTION}

Although the majority of oral sensory complaints are due to diseases restricted to the mouth, there are circumstances when such complaints are manifestations of systemic alteration. ${ }^{1}$ Menopause is a normal, developmental stage in a woman's life, which occurs at around the age of 50. It is accompanied by a number of characteristic physical changes, some of which are seen in the oral cavity. ${ }^{2}$ Oral symptoms such as dryness, burning sensation and altered taste have been reported; however, these findings are not yet fully understood. ${ }^{1}$ Dentists are generally the first healthcare professionals for patients with taste disorders, which are defined as 'diminished discrimination of the primary taste qualities or the presence of dysgeusias. ${ }^{3}$

${ }^{1 *}$ Research Assistant, ${ }^{2}$ Research Assistant, ${ }^{3}$ Associate Professor, Ankara University, Faculty of Dentistry, Department of Oral and Maxillofacial Surgery, Turkey, ${ }^{4}$ Professor, Ankara University, Faculty of Dentistry, Department of Basic Dental Sciences, Division of Biochemistry, Turkey.

${ }^{*}$ Correspondence to: Dr. Cagri Delilbasi, Research student, Osaka University, Faculty of Dentistry, First Department of Oral and Maxillofacial Surgery, 1-8 Yamadaoka, Suita, Osaka 565-0871 Japan

E-mail:delilbasi@hotmail.com

\section{Refereed paper}

Received 04.02.02; Accepted 09.08.02

(๑) British Dental Journal 2003; 194: 447-449
The aim of this study is to investigate the possible alteration of the gustatory function in postmenopausal women.

\section{SUBJECTS AND METHODS}

Forty patients admitted to Ankara University Faculty of Dentistry, Department of Oral and Maxillofacial Surgery for routine dental treatment, were included in this study. Of the total subjects, 20 were women (mean age 59.5 (SD 2.1) and a range of 53 to 70 years) and 20 were age-matched men (mean age 60.4 (SD 2.3 ) and a range of 56 to 75 years). Female subjects were selected randomly from those routinely referred to the clinic for oral health examination by Ankara University Faculty of Medicine. Complete amenorrhoea time was confirmed from the patients' records in the Faculty of Medicine. Male subjects were recruited randomly among volunteer patients. All patients taking part in the study were required to fulfil the following criteria: not be taking any medication (including hormone replacement therapy for female patients), have good oral hygiene, not have any dental or systemic disease and history that could affect gustatory function. ${ }^{3}$ All measurements were recorded in the late morning or afternoon, at least one hour after each patient had finished eating or smoking. Dentures, if worn, were removed immediately before the test.

To test gustatory function, two tests were carried out: a whole-mouth, above threshold test and a spatial (localized) taste test. For these tests sucrose, sodium chloride, citric acid and quinine hydrochloride solutions were used. Informed consent was obtained from all the participants.

\section{Whole-mouth, above threshold taste test}

For this test, the patient was instructed in the use of magnitude estimation to rate the intensity of each stimulator solution. Five concentration levels (in $1 / 2 \log$ steps) of sodium chloride $(0.01$ $\mathrm{mol} / \mathrm{L}-1.0 \mathrm{~mol} / \mathrm{L})$, citric acid $(0.32 \mathrm{mmol} / \mathrm{L}-0.032 \mathrm{~mol} / \mathrm{L})$, quinine hydrochloride $(0.01 \mathrm{mmol} / \mathrm{L}-1.0 \mathrm{mmol} / \mathrm{L})$ and sucrose $(0.01$ $\mathrm{mol} / \mathrm{L}-1 \mathrm{~mol} / \mathrm{L}$ ) were prepared in $5 \mathrm{ml}$ samples, which the patient sipped and then expectorated. The solutions were given in increasing concentrations respectively. The patient was then asked to identify the quality (salty, sour, sweet, bitter, or tasteless) and intensity of each test solution. The threshold of each solution was determined. ${ }^{4,5}$ 

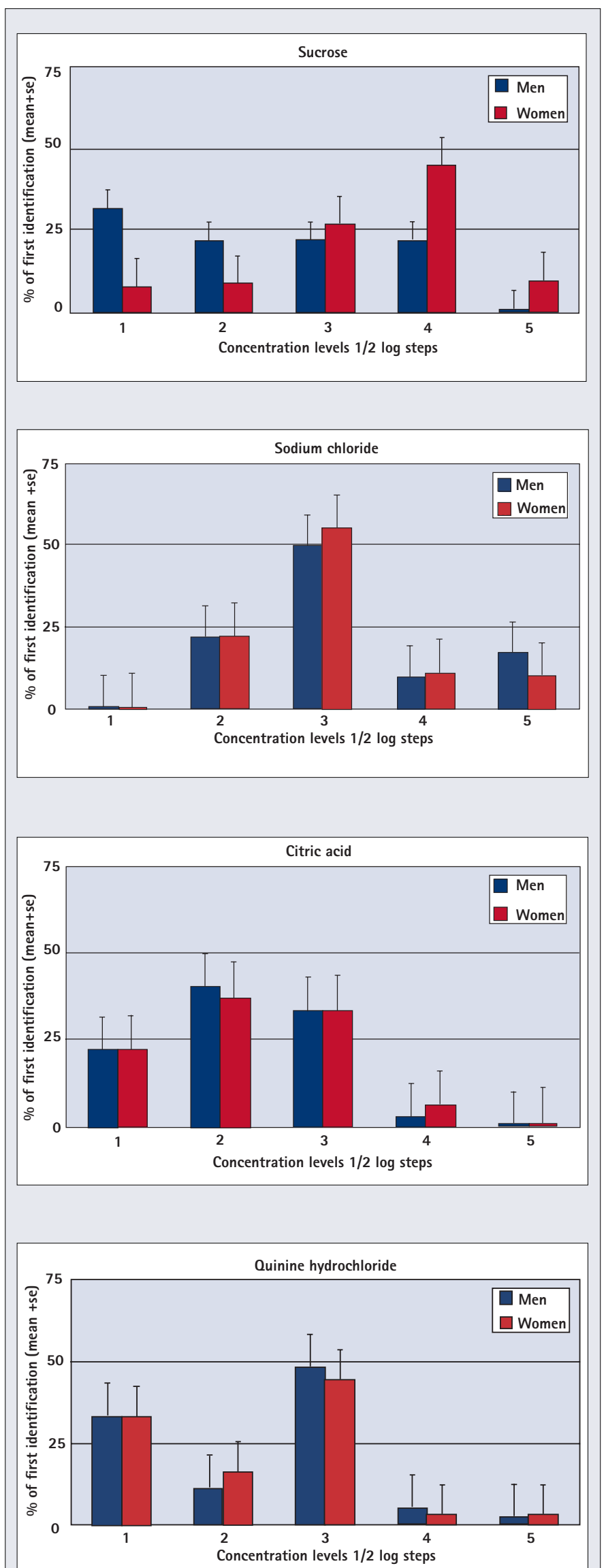

Figure 1 Percentage of correct taste quality identifications made by the patients in whole-mouth taste test. A significant difference in the perception of sucrose was found between postmenopausal women and age-matched men.

\section{Spatial (localized) taste tests}

Each patient was also tested on localized taste function. This test consists of identifying the quality of each test stimulus and rating the stimulus on an intensity scale from 0 (no taste) to 9 (very strong taste). In each trial, the strongest concentration of one of the four compounds used in the whole-mouth taste test was painted with a cotton swab on one of six locations in the mouth: the right and left anterior and posterior-lateral surfaces of the tongue (within the receptive field of the chorda tympani-lingual nerve) and the two sides of the soft palate, lateral to midline (within the receptive field of the palatine nerve). ${ }^{4,6}$

To prevent any bias in both tests, the order in which each solution was to be taken, and the order of the localizations for painting were determined randomly.

We also asked the female patients: 'Can you taste sweet, salty, sour and bitter substances as strongly as before menopause?' and 'After the menopause, did your dietary habits change?'

All the tests were done at the same visit, and to minimize fatigue or inattention, all participants were allowed to take periodic rest breaks. Measurements were taken by the two observers (Dr. Delilbasi and Dr. Cehiz). Each patient was studied by only one observer.

\section{Statistical analysis}

Mann-Whitney $U$ test or ANOVA was used with acceptance of a probability of $\mathrm{p}<0.05$ as significant.

\section{RESULTS}

Complete amenorrhoea time varied from 2 to 15 years with a mean of 9 years. There was a statistically significant difference in mean sucrose scores for the whole-mouth test $(\mathrm{P}=0.015)$, and there was a significant reduction in right $(\mathrm{P}=0.029)$ and left $(\mathrm{P}=0.03)$ palatal scores in postmenopausal women. There was no significant difference between the groups considering mean sodium chloride $(\mathrm{P}=0.991)$, citric acid $(\mathrm{P}=0.615)$ and quinine hydrochloride $(\mathrm{P}=0.39)$. Similarly, there was no significant difference in mean scores of the left anterior tongue, right anterior tongue, right posterior tongue and left posterior tongue $(\mathrm{P}=0.571$, $\mathrm{P}=0.558, \mathrm{P}=0.505$ and $\mathrm{P}=0.869$ respectively). The results are given in Figure 1 and Figure 2. Seven (35\%) of the female patients noticed failure in tasting sweet, salty, sour, and bitter substances as strongly as before menopause and 9 (45\%) reported a change in dietary habits; all indicating a preference for sweeter food.

\section{DISCUSSION}

Though menopause has long been associated with certain physical changes, the exact aetiology and the mechanisms involved in the onset of these symptoms remain unknown. ${ }^{2}$ Wardrop et al. ${ }^{1}$ found that the prevalence of oral discomfort in postmenopausal women was $46 \%$, whilst only $6 \%$ of premenopausal women experienced oral discomfort. Eighty percent of burning mouth syndrome (BMS) patients are postmenopausal women whose pain began from three years before to twelve years after the onset of menopause. It is well recognized that oral discomfort reported in menopausal women may have a number of different causes and may not be related to the hormonal changes that occur. ${ }^{1}$ Threshold tests are very valuable in olfactory testing when there is consistency in thresholds in the normal population. However, this is not the case with taste, for which there is a wide variability in normal thresholds. Taste testing of a substantial number of subjects has also been hindered by a lack of efficient and portable testing procedures. ${ }^{3,7}$

Although most taste buds are found on the dorsum of the tongue, many are also located on the soft palate, uvula, larynx, pharynx, epiglottis and oesophagus. ${ }^{3}$ At the present stage, it is 


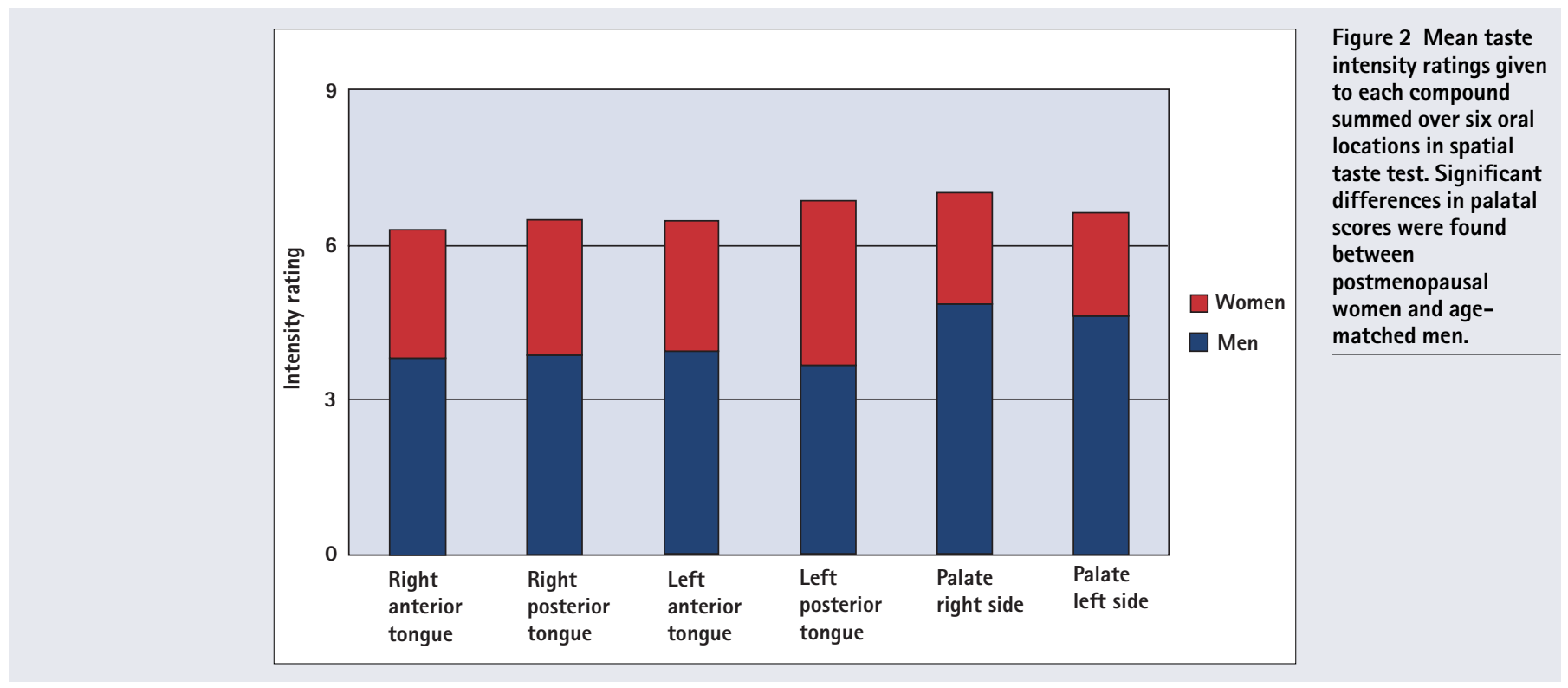

difficult to explain why there was a deficit in palatal sensation in postmenopausal women. Thus, further studies are needed to investigate oral sensory disturbance in menopause.

Age-matched male patients were selected instead of young premenopausal women as a control group in order to rule out

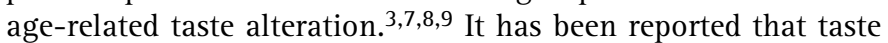
sensitivity for the basic tastes of sour, sweet, salty and bitter is greater in women than in men. ${ }^{7,8,10}$ However, since the trial findings are controversial, ${ }^{11,12,13}$ the insignificant results of sodium chloride, citric acid and quinine hydrochloride in the whole mouth test in our study are not enough to conclude that there is a decline in sensitivity in postmenopausal women. On the other hand, the decline in sensitivity to sucrose is consistent with the altered diet reported by the female patients. This finding is interesting as no previous report about failure of sucrose perception , and thus preference for sweeter food in menopausal women could be found.

Nutritional status is important in menopause because nutritional condition may have an effect on chemosensory function and changes in dietary habits may result from chemosensory dysfunction. Individuals with loss of sensitivity to sweet tastes may sweeten foods with potentially serious consequences, especially for those with diabetes mellitus, cardiac disease or obesity.

The dental practitioner can expect to encounter increasing numbers of postmenopausal women in parallel to longer life expectancies. The crucial issue to be aware of is that the possible changes due to menopause can lead to more serious health prob- lems, although these changes may not be uncomfortable to the patient. Therefore, the dental practitioner can play a very important role in the health management of postmenopausal women by recognizing and treating the oral complications of menopause.

1. Wardrop R W, Hailes J, Burger H, Reade P C. Oral discomfort at menopause. Oral Surg Oral Med Oral Pathol 1989; 67: 535-540.

2. Zachariasen R D. Oral manifestations of menopause. Compendium 1993; 14 1586-1591.

3. Mott A E, Grushka M, Sessle B J. Diagnosis and management of taste disorders and burning mouth syndrome. Dent Clin North Am 1993; 37: 33-71.

4. Shafer D M, Frank M E, Gent J F, Fisher M E. Gustatory function after third molar extraction. Oral Surg Oral Med Oral Pathol Oral Radiol Endod 1999; 87: 419-428.

5. Bartoshuk L M, Gent J, Catalanotto F A, Goodspeed R B. Clinical evaluation of taste. Am J Otolaryngol 1983; 4: 257-260

6. Bartoshuk L M, Catalonatto FA, Scott A E, Solomon G M. Spatial taste associated with head trauma, upper respiratory infection and nasal symptoms. Chem Senses 1989; 14: 684-687.

7. Mojet J, Hazelhof E C, Heidema J. Taste perception with age: Generic or specific losses in threshold sensitivity to the five basic tastes? Chem Senses 2001; 26: 845860.

8. Matsuda T, Doty R L. Regional taste sensitivity to $\mathrm{NaCl}$ : relationship to subject age, tongue locus and area of stimulation. Chem Senses 1995; 20: 283-290.

9. Fikentscher R, Rosenburg B, Spinar $H$, Bruchmuller W. Loss of taste in the elderly: sex differences. Clin Otolaryngol 1977; 2: 183-189.

10. Velle W. Sex differences in sensory functions. Persp Biol Med 1987; 30: 490-522.

11. Hyde R J, Feller R P. Age and sex effects on taste of sucrose, $\mathrm{NaCl}$, citric acid and caffeine. Neurobiol Aging 1981; 2: 315-318.

12. Murphy C, In Hall S S, Coons D H. The effects of age on taste sensitivity. Special senses in aging. University of Ann Arbor, Institute of Gerontology, Ann Harbor, MI pp 21-33, 1979.

13. Stevens J C, Cain W S, Demarque A, Ruthruff A M. On the discrimination of missing ingredients: aging and salt flavour. Appetite 1991; 16:129-140. 